\title{
THE EMERGENCE OF COHERENT WAVE GROUPS IN DEEP-WATER RANDOM SEA
}

\author{
CLAUDIO VIOTTI, DENYS DUTYKH*, JOHN M. DUDLEY, AND FRÉDÉRIC DIAS
}

\begin{abstract}
Extreme surface waves in deep-water long-crested sea are often interpreted as a manifestation in real world of the so-called breathing solitons of the focusing nonlinear Schrödinger equation. While the spontaneous emergence of such coherent structures from nonlinear wave dynamics was demonstrated to take place in fiber optics systems, the same point remains far more controversial in the hydrodynamic case. With the aim to shed further light on this matter, the emergence of breather-like coherent wave groups in longcrested random sea is here investigated by means of high-resolution spectral simulations of the fully nonlinear two-dimensional Euler equations. The primary focus of our study is to parametrize the structure of random wave fields with respect to the Benjamin-Feir index, which is a nondimensional measure of the energy localization in Fourier space. This choice is motivated by previous results, showing that extreme-wave activity in long-crested sea is highly sensitive to such parameter, which is varied here by changing both the characteristic spectral bandwidth and the average wave steepness. It is found that coherent wave groups, closely matching realizations of Kuznetsov-Ma breathers in Euler dynamics, develop within wave fields characterized by sufficiently narrow-banded spectra. The characteristic spatial and temporal scales of wave group dynamics, and the corresponding occurrence of extreme events, are quantified and discussed by mean of space-time autocorrelations of the surface elevation envelope and extreme events statistics.
\end{abstract}

Key words and phrases: Breathers; random seas; coherent structures; extreme waves

\section{Contents}

\section{Introduction}

2 Governing equations and numerical simulations 3

2.1 Random initial data

\section{Results}

3.1 Autocorrelations

3.2 Extreme-event statistics

3.3 Effect of wave steepness

2010 Mathematics Subject Classification. 76B15 (primary), $76 \mathrm{~B} 07$ (secondary).

* Corresponding author. 


\section{Introduction}

Surface waves of outstandingly high amplitude, often referred to as "rogue waves", can occur spontaneously in the ocean. Nowadays the existence of such extreme events is a well documented fact $[5,21,22]$, and there is widespread awareness that rogue waves represent a concrete hazard for ships and marine structures [1]. At the same time, however, rogue waves remain poorly understood phenomena despite the intense research efforts carried by the scientific community, thereby representing an open and intriguing subject of study. The term "rogue wave" is often used quite generically in referring to extreme waves caused by different mechanisms and even pertaining to different physical natures. In the case of oceanic surface waves, specific factors such as bottom bathymetry, currents or wind, can trigger the formation of extreme events in different ways (see the review papers by KHARIF \& Pelinovsky [16] and Dysthe et al. [8]). Moreover, analogous rogue wave phenomena also belong to the realm of nonlinear optics $[11,17,18]$. In this study we shall focus on extreme waves caused by the self-focussing mechanism of surface waves in deep water, furthermore, we narrow our study to the case of two spatial dimensions (i.e., infinitely long-crested waves).

Several reduced mathematical models have been developed for the study of deep-water gravity waves. Among them, the nonlinear Schrödinger (NLS) equation is perhaps the most successful in describing the two-dimensional energy self-focussing mechanism. Such mechanism is the primary candidate for explaining the spontaneous formation of extreme waves "out of nowhere", and arises from the interplay between nonlinearity and dispersion. Several exact solutions are known for the NLS equation. In particular, the so-called breathing solitons, or "breathers", have for long been regarded as analytical models of rogue waves $[9,13,26]$. We remark that such mathematical framework has proven effective in the optics context: recently, KIBLER et al. [18] have provided the first experimental evidence that extreme fluctuations in fiber-optics systems are associated with formation of NLS breathers, while GENTY et al. [11] have shown that breathers interactions are effective in describing the nonlinear processes leading to optical rogue waves.

In order to assess the realm of validity of breather dynamics in the context of deep water free-surface waves, different authors have explored the survival of such structures under Euler dynamics, both in numerical computations [13] and, more recently, in wave-tank experiments $[3,2]$. Altogether, such studies have shown that breathers are quite robust even in the realm of fully nonlinear hydrodynamic waves, at least for relatively small wave steepness and sufficiently short time scales. In the most part of previous investigations, however, breathers were created within the background wave field by mean of properly devised setups, and little is known about their likelihood to emerge spontaneously in random sea states. Remarkably, this important aspect of the problem has been addressed only in a few instances $[24,23,30]$ and limited to the framework of the NLS equation. We also note that, in order to shed light on the structures embedded in a random sea, it is necessary to follow the evolution of wave groups over large spatial and temporal scales jointly. As also other authors remark [27], this represents a challenging task for laboratory and field investigations, whereby less information is available in regard to this particular aspect of extreme-wave dynamics. 
In regard to the study of random sea states, several investigations have focused on collecting statistics of extreme wave events. Also in this case results are available from both laboratory experiments [25, 29] and numerical simulations [15, 33, 12, 28, 32, 36]. A central finding of such studies consists of the intensification of extreme wave activity for high values of the Benjamin-Feir index (see $\S 2.1$ ), i.e., when the power spectrum of the surface elevation is sharply peaked around the fundamental wavenumber. Other recent studies [34] suggest that mechanisms may exist in the ocean capable of leading to narrow-banded spectra, therefore making this kind of regime of potential interest for oceanic waves. Narrow-banded spectra correspond to slowly modulated wave fields in physical space, in this condition modulational (or Benjamin-Feir, or side-band) instability arises and develops until a nonlinear saturation is attained [33, 32]. As a result of such an instability, the spectral band-width broadens, and the associated value of the BenjaminFeir index decreases below its critical value. This fact, together with observations of high wave amplifications occurring during the disruption of uniform wave trains undergoing Benjamin-Feir instability [6], underpins the idea that a connection exists between rogue waves and Benjamin-Feir instability. Different authors [33, 37, 32] have suggested that rogue waves are a result of such transient evolution.

If extreme waves are to be considered a consequence of breathing modes embedded in a random background [25], then the increased probability of extreme waves for narrowbanded spectra should reflect into a stronger presence of organized structures within the wave field. This is the main point under investigation in this paper, which is organized as follows. In $\S 2$ we describe the mathematical setup of our study, including governing equations and initial conditions. In $\S 3$ we present and discuss our main numerical results, we have organized this section into a first part, §3.1, which is focused on the description of the physical structure of the wave field, and a second part, §3.2, which contains global statistics related to extreme wave events, and §3.3, which addresses the effect of wave steepness.

\section{Governing equations and numerical simulations}

We perform direct numerical simulations of the free-surface Euler equations in two spatial coordinates $(x, y)$ and time $t$. We employ periodic boundary conditions in the horizontal $(x)$ direction. By assuming irrotational flow, and neglecting surface tension effects, the free surface $\eta(x, t)$ and the velocity potential $\phi(x, y, t)$ are governed by the system

$$
\begin{aligned}
& \left.\begin{array}{l}
\eta_{t}=-\phi_{x} \eta_{x}+\phi_{y}, \\
\phi_{t}=-\frac{1}{2}\left(\phi_{x}{ }^{2}+\phi_{y}{ }^{2}\right)-g \eta,
\end{array}\right\} \quad \text { at } \quad y=\eta(x, t) \\
& \nabla^{2} \phi=0, \quad \text { for } \quad-\infty<y<\eta(x, t)
\end{aligned}
$$

where $g$ is the gravity acceleration. (The fluid density is assumed here to be unitary without loss of generality.) Under the deep water approximation the potential $\phi$ must satisfy the 
boundary condition

$$
\lim _{y \rightarrow-\infty}|\nabla \phi|=0 .
$$

The numerical scheme we employ for solving the system (2.1) is a high-order spectral method introduced by DYACHENKo et al. [7], which is based on the conformal transformation that maps the time-dependent flow domain into the lower-half complex plane. We do not report here the equations resulting from the conformal mapping (these can be found, e.g., in Choi \& Camassa [4] or Milewski et al. [19]). In the following two sections we describe in detail the initial conditions employed in this study. The values set for the main numerical parameters are reported in $\S 3$.

\subsection{Random initial data}

In this set of simulations we initialize the Fourier coefficients of $\eta$ and $\bar{\phi}$ as

$$
\hat{\eta}_{k}=\left[2 P_{0}(k)\right]^{1 / 2} e^{i k \varphi_{k}}, \quad \hat{\phi}_{k}=i c_{k} \hat{\eta}_{k},
$$

with $k=2 \pi n / L, n=0,1, \ldots N$. The function $P_{0}(k)$ is the prescribed potential-energy power spectrum and the $\varphi_{k}$ 's are independent, uniformly distributed random phase taking values in the interval $[0,2 \pi]$. We link the potential at the free surface to the surface displacement using the phase velocity, $c_{k}=\sqrt{g / k}$, as for linear traveling waves. This choice determines an overall propagation speed for the random wave field, and implies an initial equipartition of the total energy of the wave field in potential and kinetic energy. Such equipartition remains substantially preserved during the ensuing time evolution. We use the Gaussian initial spectrum

$$
P_{0}(k)=\frac{\mathcal{P}_{0}}{\sqrt{2 \pi} \sigma_{0}} \exp \left[-\frac{1}{2}\left(\frac{k-k_{0}}{\sigma_{0}}\right)^{2}\right],
$$

which depends on the parameters $\mathcal{P}_{0}, k_{0}$ and $\sigma_{0}$, respectively the total (initial) potential energy, peak wavenumber and spectral width.

Another important parameter characterizing the spectrum of the surface elevation at a fixed time, $P(k, t)$, is the Benjamin-Feir index, BFI(t). Following Janssen [15], we define this parameter as

$$
\mathrm{BFI}=\sqrt{2} \mathrm{~s} \frac{\mathrm{k}_{\mathrm{w}}}{\sigma_{\mathrm{w}}}
$$

with spectral width and characteristic wavenumber respectively given by

$$
\sigma_{w}=\frac{\int_{0}^{+\infty}\left(k-k_{w}\right)^{2} P \mathrm{~d} k}{\int_{0}^{+\infty} P \mathrm{~d} k}, \quad k_{w}=\frac{\int_{0}^{+\infty} k P \mathrm{~d} k}{\int_{0}^{+\infty} P \mathrm{~d} k}
$$

and wave steepness $s=k_{w} \eta_{\mathrm{rms}}$, where

$$
\eta_{\mathrm{rms}} \equiv\left\langle\eta^{2}\right\rangle^{1 / 2}=2\left\langle\int_{0}^{+\infty} P \mathrm{~d} k\right\rangle^{1 / 2}
$$

The above definition holds for general spectral shapes, therefore it is suitable for timeevolving spectra. Initially, we have clearly that $k_{w} \simeq k_{0}$, which essentially remains true for all times, and $\sigma_{w} \simeq \sigma_{0}$, which on the contrary does not hold during time evolution. Note 
that when the spatial spectrum is considered, as opposed to the temporal spectrum $P(\omega)$ (more suitable for experimental measurements), the corresponding value of BFI converts to roughly one half of its temporal counterpart. This simply follows from the fact that $\mathrm{d} k / \mathrm{d} \omega \simeq 2 / \sqrt{\frac{k_{0}}{g}}$ for $k$ close to $k_{0}$, according to the linear dispersion relation $\omega=\sqrt{g k}$.

Since BFI is time-dependent, we distinguish between two ways of defining its characteristic value. We consider the initial value

$$
\mathrm{BFI}_{0}=\operatorname{BFI}(0)
$$

and the time-average value

$$
\langle\mathrm{BFI}\rangle=\lim _{\mathrm{T} \rightarrow+\infty} \frac{1}{\mathrm{~T}} \int_{0}^{\mathrm{T}} \operatorname{BFI}(\mathrm{t}) \mathrm{dt}
$$

When $\mathrm{BFI}_{0}$ is above a critical value (about 0.5 ) the two above definitions differ significantly, as a result of Benjamin-Feir instability.

\section{Results}

We set the parameters of the initial spectrum (2.2) in such a way to span a range of values of $\mathrm{BFI}_{0}$, while keeping the wave steepness $s$ constant. We employ a domain size, $L$, much larger that the typical wave length $\lambda_{0}=2 \pi / k_{0}$, but also larger than the scale of the initial modulation, which is significantly larger than $\lambda_{0}$ for small values of $\sigma_{0}$. Namely, we set $L=124 \pi$ up to $L=512 \pi$ for the highest values of $\mathrm{BFI}_{0}$ considered. The effective number of Fourier modes we use is $N=2^{14}$ up to $N=2^{16}$ after dealiasing, which corresponds to a maximum wave number equal to $256 k_{0}$. This resolution was always sufficient to represent the power spectrum up to decay below machine precision. Hereinafter, spatial and temporal units shall be normalized on $k_{0}$ and $\sqrt{g k_{0}}$ respectively.

The largest domain considered here, $L=512 \pi$, contains about 128 waves (in a crest-tocrest count), while the largest wave tanks employed in recent experimental studies $[25,28]$ allow to accommodate about 60 waves (for the wavelength employed). In the typical experimental setup, however, waves are generated on one side of the tank by a wavemaker, so that the effective spatial extension corresponding to a time signal recorded at a fixed location in space is given by $L_{e}=c_{g} T_{e}$, where $T_{e}$ is the entire duration of the experiment. The length scale $L_{e}$ is the proper analogue of the domain size in our numerical simulations. On the other hand, the effective time scale observed in experiments, in terms of the observable evolution of individual wave groups, is set by the time a wave group takes to propagate from the wave-maker to the opposite end of the tank. This time scale is the analogue of our final simulation time $T$. The experiments by OnORATO et al. [25], and similarly those by SHEMER et al. [28], are effectively equivalent to a numerical simulation with $L \approx 2300$ and $T \approx 550$, hence they contain a larger spatial sample but a much smaller amount of time evolution.

To begin with, we can observe the qualitative features of the time evolution of the wave field from random initial data in the visualizations reported in Figure 1 (left panels). In these pictures we report the wave envelope evolution for different values of $\mathrm{BFI}_{0}$. The wave 
envelope, denoted as $\xi(x, t)$, is defined by [14]

$$
\xi=\sqrt{\eta^{2}+\tilde{\eta}^{2}}
$$

where $\tilde{\eta}$ is the Hilbert transform of $\eta$ :

$$
\tilde{\eta}(x, t)=\int_{-\infty}^{\infty} \frac{\eta\left(x^{\prime}, t\right)}{x-x^{\prime}} \mathrm{d} x^{\prime} .
$$

The envelope is a natural mean to identify wave groups within a general two-dimensional wave field [14], indeed in the frame of reference traveling at the group velocity, $c_{g}=\frac{1}{2} \sqrt{g / k_{0}}$ a first visual inspection (Figure 1) may not reveal any overall propagation direction of wave groups. The sequence of visualizations reported in Figure 1 shows the strong dependence of the physical scales on the initial spectral distribution. In particular, large amplitude events appear progressively more organized in long-lived coherent structures as $\mathrm{BFI}_{0}$ increases. The fully developed coherent structures (shown also later, more prominently, in Figure 3) closely resemble analogous visualizations by GENTY et al. [11] of simulated breather dynamics in a class of generalized NLS equations. Note that very large events $\left(\xi \gtrsim 4 \eta_{\mathrm{rms}}\right.$, say) are present in all four cases reported in Figure 1, even though this detail is perhaps harder to appreciate in the uppermost panel $(a)$. The trend described can be interpreted qualitatively within the framework of the NLS equation. Indeed, upon rescaling variables in the dimensional NLS equation as

$$
A^{\prime}\left(x^{\prime}, t^{\prime}\right)=s k_{0} A\left(\frac{x}{\ell}, \frac{t}{\tau}\right)
$$

where $A$ is the customary dependent variable (the complex envelope), $\ell$ is the characteristic spatial scale of the initial data, and $\tau \equiv\left(k_{0} \ell\right)^{2} / \omega_{0}$, one obtains a non dimensional NLS equation in which the Benjamin-Feir index, given by $s k_{0} \ell$, appears as the coefficient of the nonlinear term (the other coefficients being constants). This motivates the generally adopted interpretation of such parameter as a measure of the relative strength of nonlinearity versus linear dispersion, the second appearing to be responsible for depleting coherent structures.

\subsection{Autocorrelations}

In order to quantify the characteristic spatial and temporal scales of wave groups we employ the envelope space-time autocorrelation function:

$$
C_{x t}(\Delta x, \Delta t) \equiv \frac{\langle\tilde{\xi}(X, t) \tilde{\xi}(X+\Delta x, t+\Delta t)\rangle}{\left\langle\tilde{\xi}^{2}\right\rangle},
$$

where $\tilde{\xi} \equiv \xi-\langle\xi\rangle$. According to the customary definition of autocorrelation functions, $C_{x t}$ is here normalized on the variance $\left\langle\tilde{\xi}^{2}\right\rangle$, whereby $C_{x t}(0,0)=1$. Also in this case we work in the traveling frame of reference $X=x-c_{g} t$. Autocorrelation functions are used as a mean to extract the dominant scales and morphological features of random fields. We also mention the use of two-point two-time statistics in the study of random sea based on statistical models [10]. The statistical averaging understood in the above definition includes spatial, temporal and ensemble (i.e., over several independent realizations of the same run) averaging. It should be noted that time averaging relies on the verified assumption that 
(a)

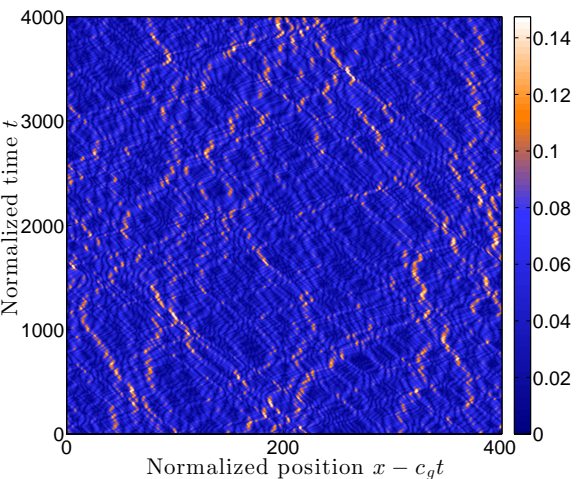

(c)

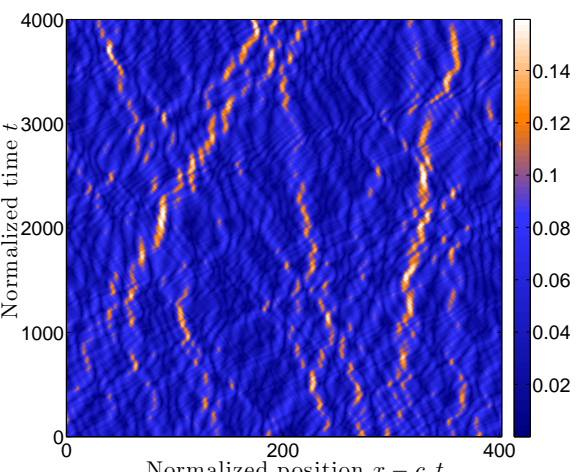

Normalized position $x-c_{g} t$

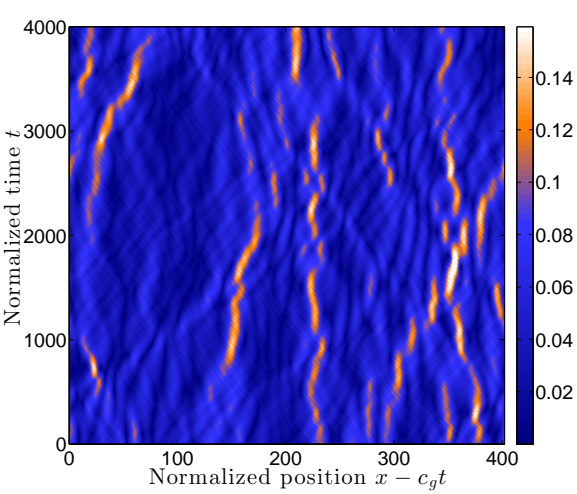

(e)

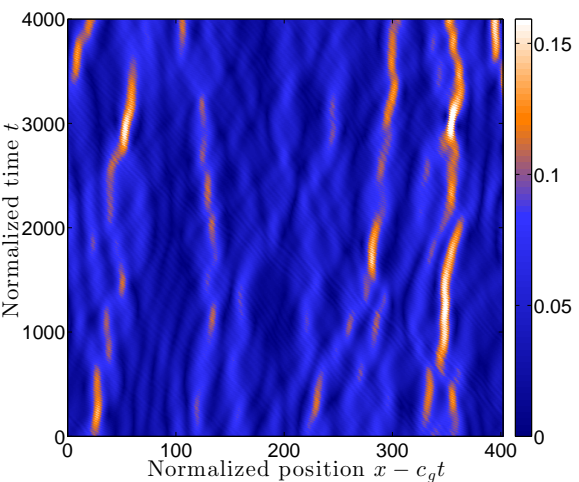

(b)

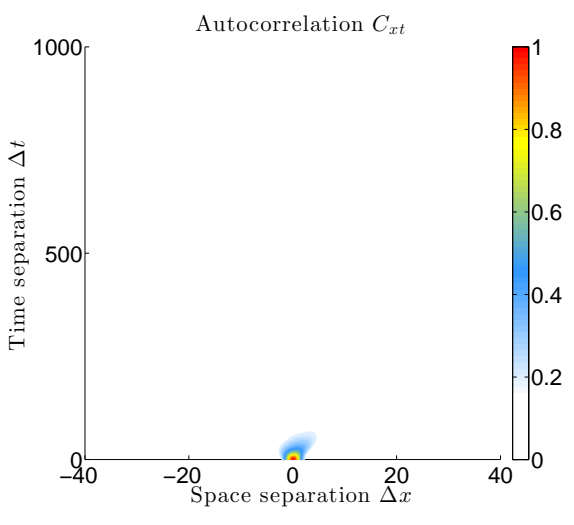

(d)

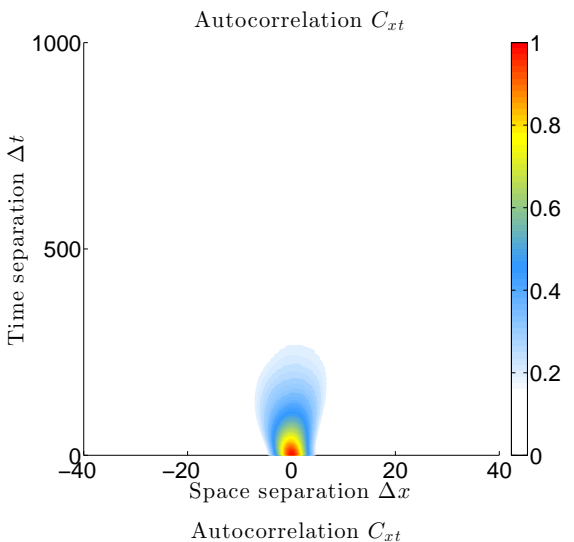

f)
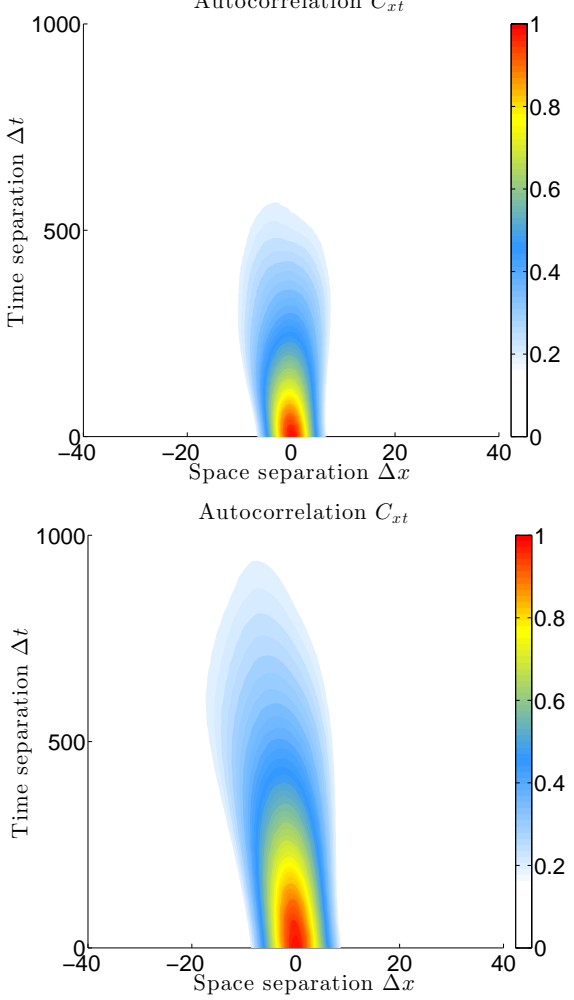

Figure 1. Time evolution of the wave envelope, $\xi$, from Euler numerical simulations (left panels) and corresponding space-time autocorrelation functions, $C_{x t}$, (right panels) for increasing values of BFI. Values of the BFI are: (a-b) 0.123, (c-d) 0.216, (e-f) 0.348, ( $g$ - $h$ ) 0.500. All four cases share the same wave steepness, $s=0.04$. Spatial and temporal units are normalized using $k_{0}$ and $\sqrt{g k_{0}}$ respectively. 


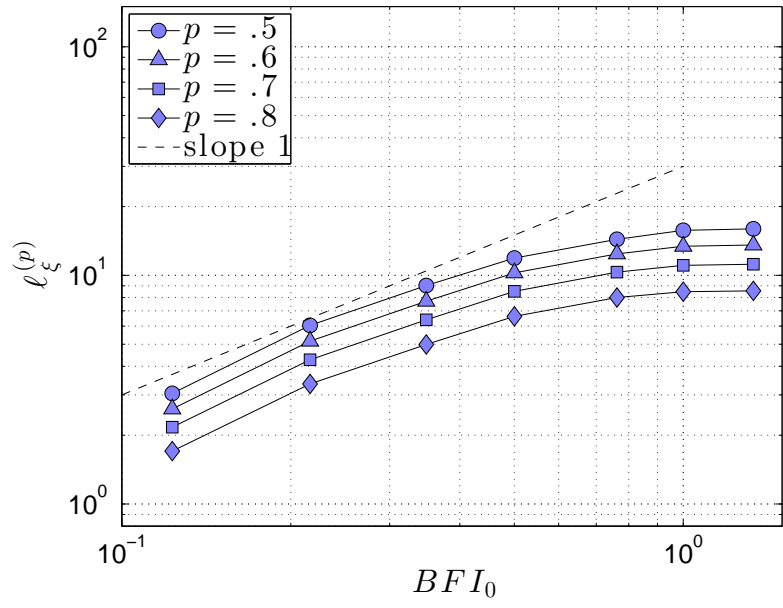

(a)

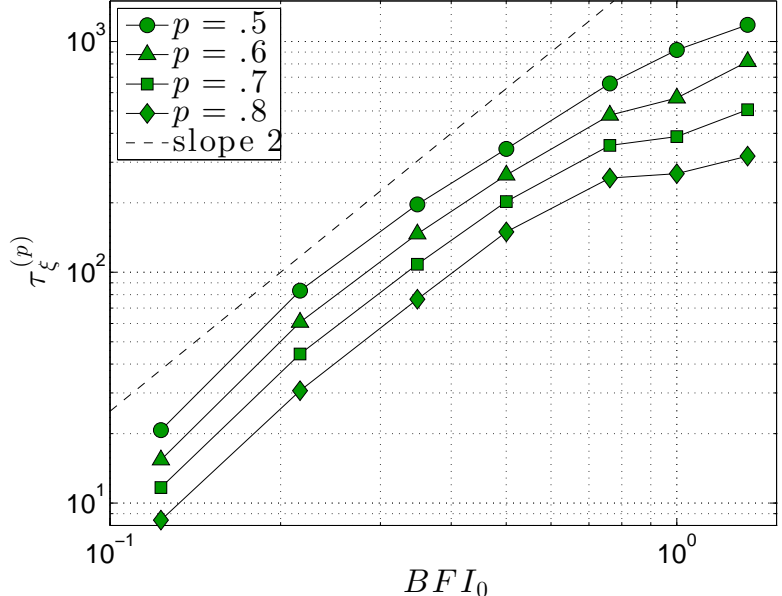

(b)

Figure 2. Spatial (a) and temporal (b) autocorrelation scales versus $\mathrm{BFI}_{0}$ from Euler numerical simulations. Dashed lines reference power-law trends.

a statistically steady state is effectively attained over the time scales considered after a faster initial transient.

The autocorrelation functions computed are reported in Figure 1 (right column) next to the corresponding field visualizations, in order to show how the emergence of coherent patterns in $(x, t)$ space reflects in the development of long-range autocorrelations. Note how, beside the evident change of the scale of $C_{x t}$, more subtle details exhibit variation across the different cases. In particular, the autocorrelation functions are not symmetric with respect to $\Delta x$, but show a preferential direction which is tilted with respect to the vertical axis in the $(\Delta x, \Delta t)$ space. This fact denotes a non-zero mean drift velocity of the wave groups in addition to the linear group velocity, which is an nonlinear effect (cf. §3.3). It is interesting to observe how such an orientation inverts from the first to the last case. In the last case (panels $(\mathrm{g})$ and $(\mathrm{h})$ ) the mean drift velocity is smaller than $c_{g}$ (roughly by one order of magnitude), yet clearly visible in the autocorrelation function. Note that leftward orientation in the $(\Delta x, \Delta t)$ plane translates into rightward-oriented structures in the $(x, t)$ plane.

Characteristic scales can be defined using the correlation function in a number of ways. Here we define the length scale, $\ell_{\xi}^{(p)}$, and the life-time scale, $\tau_{\xi}^{(p)}$, as

$$
\ell_{\xi}^{(p)}=\Delta x_{+}^{(p)}-\Delta x_{-}^{(p)}, \quad \tau_{\xi}^{(p)}=A^{(p)} / \ell_{\xi}, \quad(0<p<1)
$$

where $\Delta x_{ \pm}^{(p)}$ denotes either the positive or negative intersections between the $C_{x t}=p$ level set and the horizontal $(\Delta t=0)$ axis (i.e., $\left.C_{x t}\left(\Delta x_{ \pm}^{(p)}, 0\right)=0\right)$, while $A^{(p)}$ is the area in the $\Delta x-\Delta t$ plane enclosed within the same level set $C_{x t}=p$.

The dilation of space-time correlation scales with respect to $\mathrm{BFI}_{0}$ is summarized in Figure 2. Different values of the parameter $p$ are considered, in order to verify that the overall trend is not strongly affected by this specific choice. The results show that the 


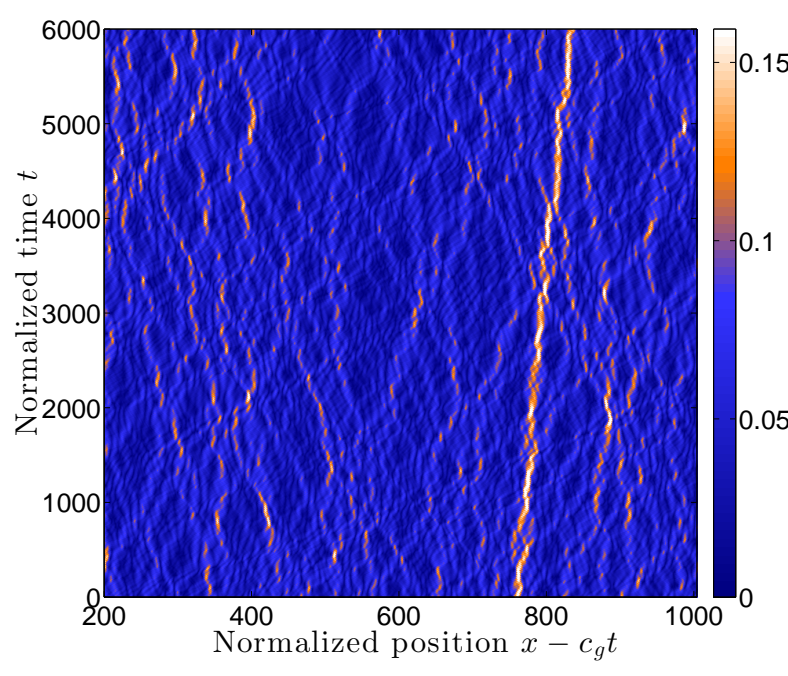

(a)

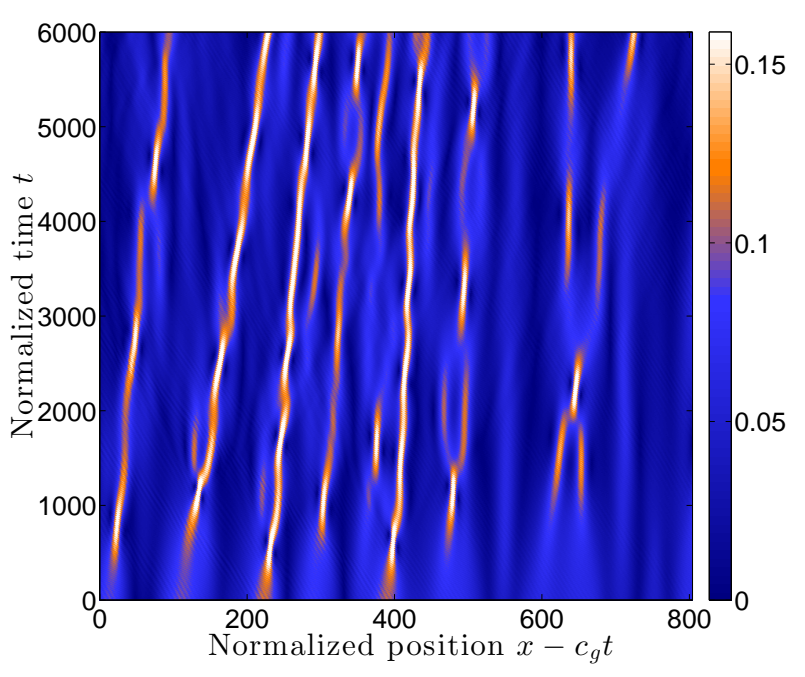

(b)

Figure 3. Coherent structures emerging in the time evolution of the wave envelope, $\xi$, from Euler numerical simulations at moderate (a) and high (b) value of the Benjamin-Feir index $\left(\mathrm{BFI}_{0}=0.216\right.$ and $\mathrm{BFI}_{0}=1.333$, respectively). Spatial and temporal units are normalized using $k_{0}$ and $\sqrt{g k_{0}}$ respectively.

growth of the spatial correlation length is milder that the corresponding growth of time correlation scale. In particular, $\ell_{\xi}^{(p)}$ follows a growth trend that is slower than linear, while $\tau_{\xi}^{(p)}$ grows at a rate closer to a quadratic power law. Note that the spatial time scale settles on a constant value once $\mathrm{BFI}_{0} \approx 1$, whereas the temporal correlation scale continues to increase within the whole range considered. This fact is consistent with the observation that further increments of the BFI, beyond a critical value, result in a stronger ordering of the distribution of coherent structures, as can be appreciated from Figure 3. We further stress that the observed variation of correlation scales does not follow from any simple scaling analysis that can be derived in the framework of the NLS equation.

For the purpose to illustrate the similarity between the coherent structures shown in Figure 3 we next show a numerical simulation initialized using a time-periodic KuznetsovMa breather solutions of the NLS equation. In terms of the complex envelope, $A$, which modulates a monochromatic carrier wave of wavenumber $k_{0}$, such solution in dimensional form reads as

$$
A\left(x^{\prime}, t^{\prime}\right)=a_{0} \frac{\cos \left(\Omega t^{\prime}-2 \mathrm{i} \varphi\right)-\cosh \varphi \cosh p x^{\prime}}{\cos \Omega t^{\prime}-\cosh \varphi \cosh p x^{\prime}} \mathrm{e}^{2 \mathrm{i} t^{\prime}}
$$

where

$$
\begin{aligned}
& x^{\prime}=\sqrt{2} a_{0} k_{0}^{2} x, \quad t^{\prime}=-\frac{1}{4} \omega_{0} k_{0}^{2} a_{0}^{2} t, \\
& \Omega=2 \sinh 2 \varphi, \quad \omega_{0}=\sqrt{g k_{0}},
\end{aligned}
$$

and $0<\varphi<+\infty$ is a non dimensional parameter. In the limit $\varphi \rightarrow 0$ the Kuznetsov-Ma breather approaches the so-called Peregrine soliton [13, 17], i.e., a single-pulse event in 


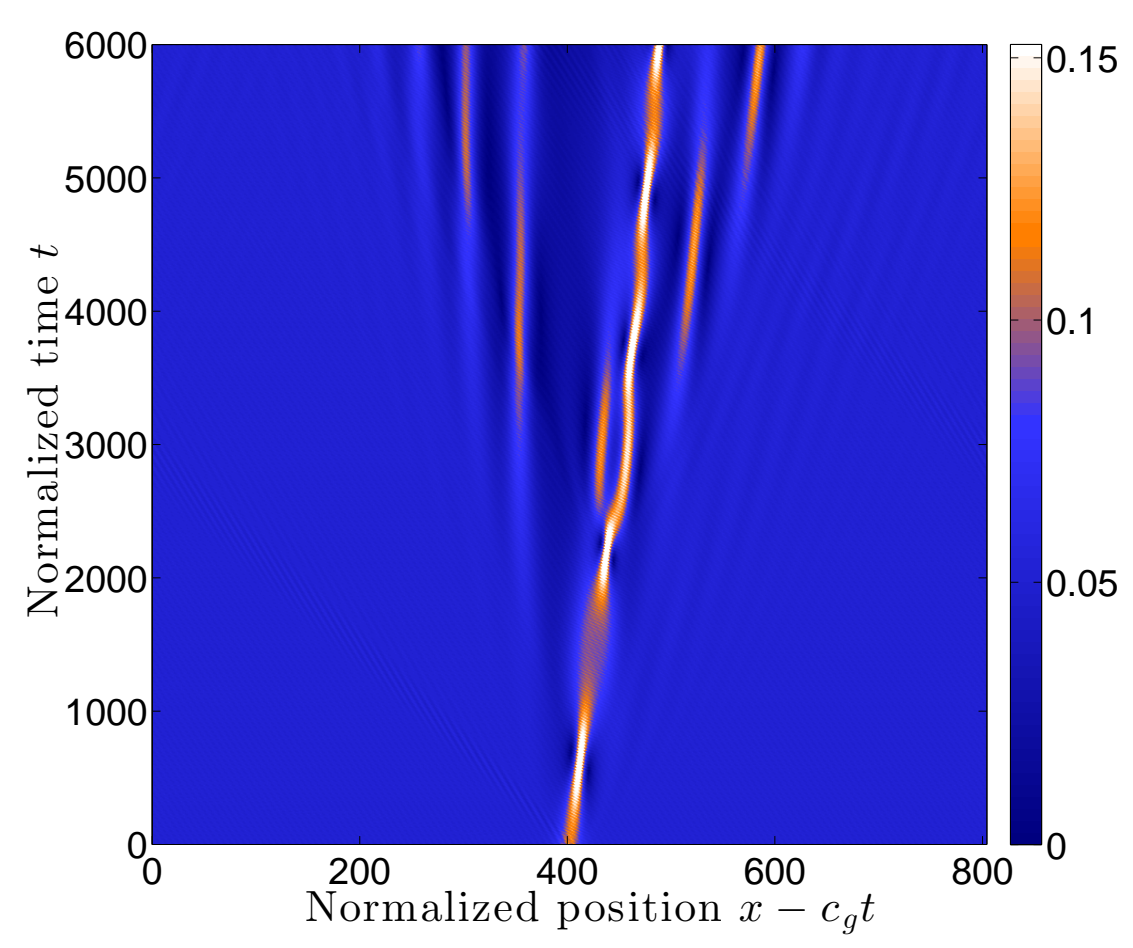

Figure 4. Time evolution of the wave envelope, $\xi$, from Euler numerical simulations for Kuznetsov-Ma breather initial condition. The period of the breather is $2 \pi / \Omega=1200$. Spatial and temporal units are normalized using $k_{0}$ and $\sqrt{g k_{0}}$ respectively.

both space and time. In order to implement the above formula as an initial condition for the Euler simulations the surface displacement and the potential at the surface are reconstructed as

$$
\eta_{0}=A\left(x^{\prime}, 0\right) \mathrm{e}^{\mathrm{i} k_{0} x}+\text { c.c. }, \quad \phi_{0}=-\mathrm{i} \frac{\omega_{0}}{k_{0}} A\left(x^{\prime}, 0\right) \mathrm{e}^{\mathrm{i} k_{0} x}+\text { c.c. }
$$

While formula (3.2) is not an exact solution for the Euler equations, the breather structure is able to survive over a long time scale, as shown in Figure 4. The comparison between Figures 3 and 4, observing amplitude and typical scales, shows how NSL breathers can effectively be thought of as analytical models for the coherent structures that emerge spontaneously from random initial data.

\subsection{Extreme-event statistics}

We present here further results aimed to illustrate how extreme-event statistics modify along with the change of structure of the wave field discussed in the previous section. Figure 5 contains the probability density functions (PDFs) of the wave envelope $\xi$ for different values of $\mathrm{BFI}_{0}$. Consistently with previous results [25], we find that PDFs develop heavy tails as the value of BFI increases. As shown in the same picture, in the cases with the largest BFI values such tails are significantly underpredicted by the canonical first- 


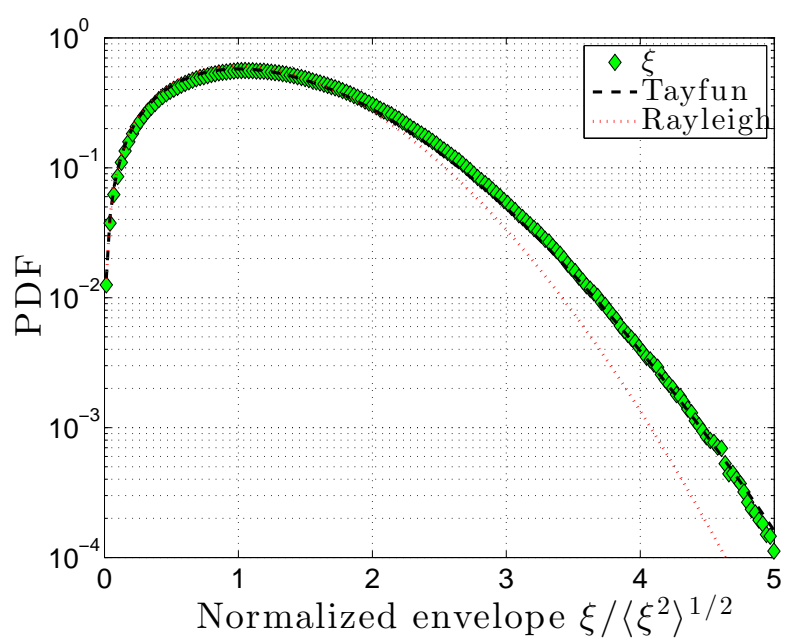

(a)

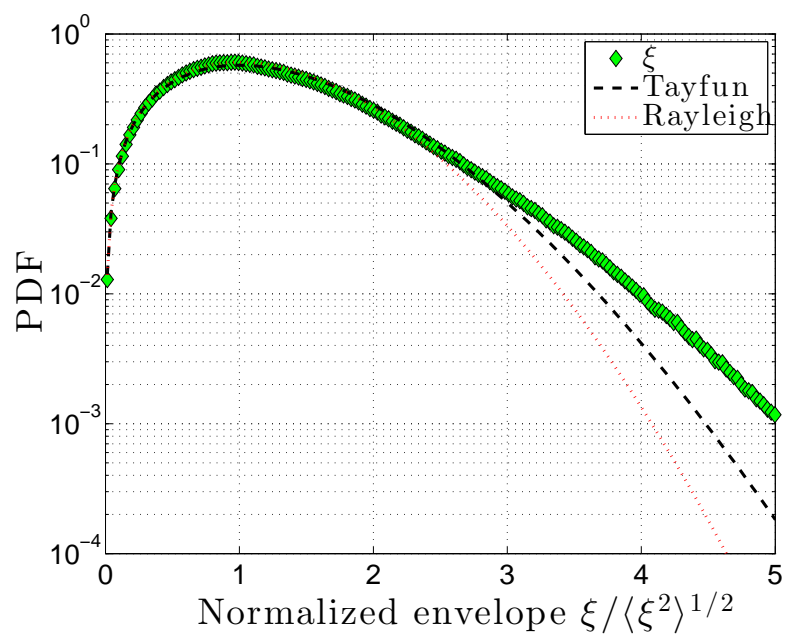

(c)

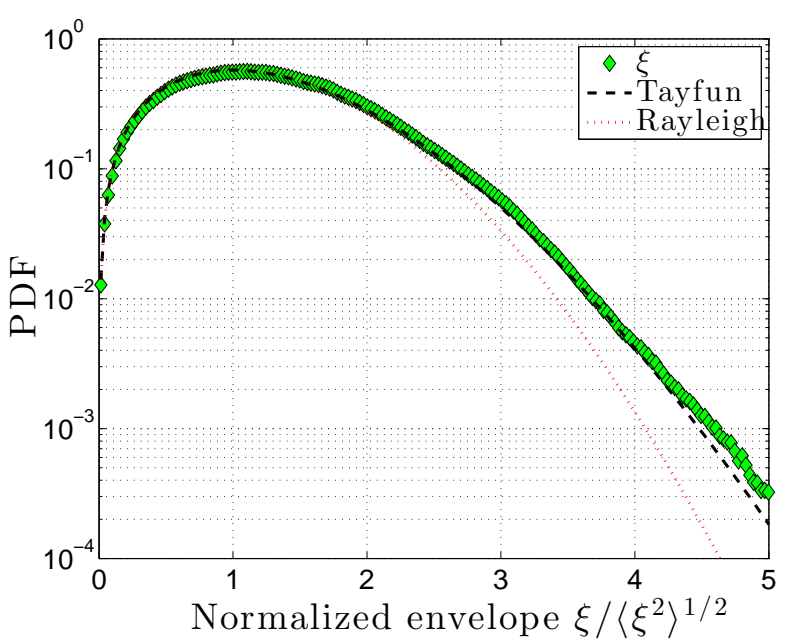

(b)

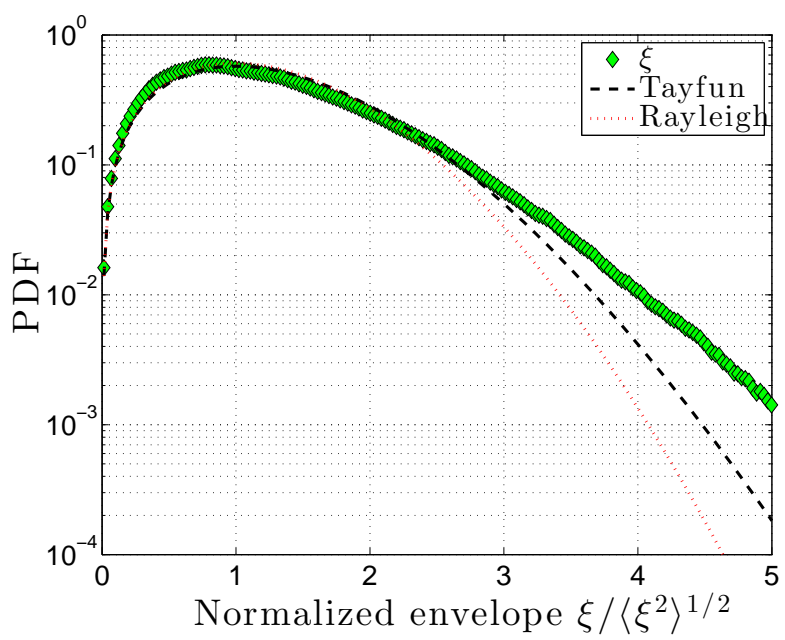

(d)

Figure 5. PDFs of the wave envelope, $\xi$, corresponding to the four cases reported in figure 1. Euler numerical simulations (symbols), Tayfun distribution (dashed lines) and Rayleigh distribution (dotted lines).

and second-order statistical models given by the Rayleigh and Tayfun [35] distributions respectively.

In Figure 6 we report the kurtosis, $\kappa$, of the surface elevation as a function of the Benjamin-Feir index. We recall that the customary definition of such a quantity is

$$
\kappa \equiv \frac{\left\langle\eta^{4}\right\rangle}{\left\langle\eta^{2}\right\rangle^{2}}-3
$$

The two displayed data sets represent the same values of $\kappa$ plotted as a function of the initial and time-averaged values of BFI respectively. The plot shows that both $\kappa$ and $\langle\mathrm{BFI}\rangle$ reach a limit with respect to $\mathrm{BFI}_{0}$. Even for subcritical initial conditions, the instantaneous value of BFI decreases during the initial stage of time evolution. The analytic prediction 


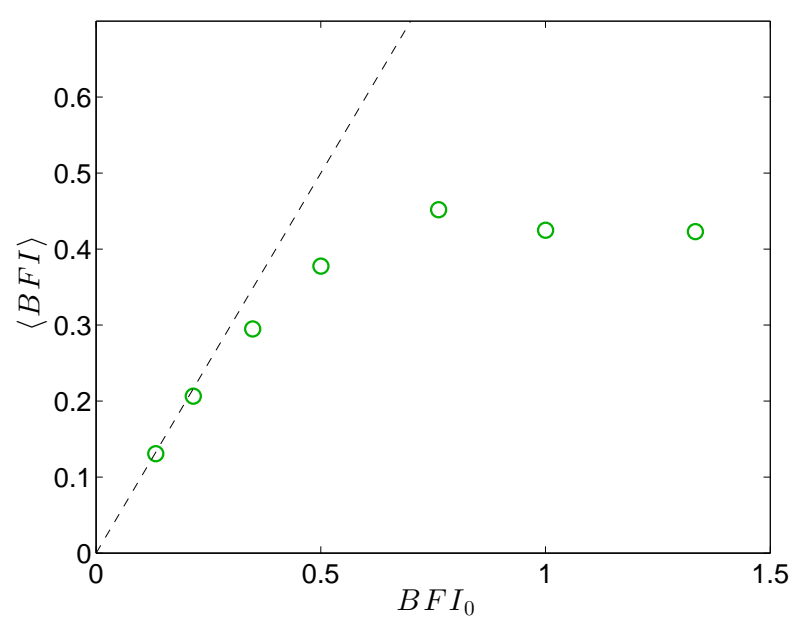

(a)

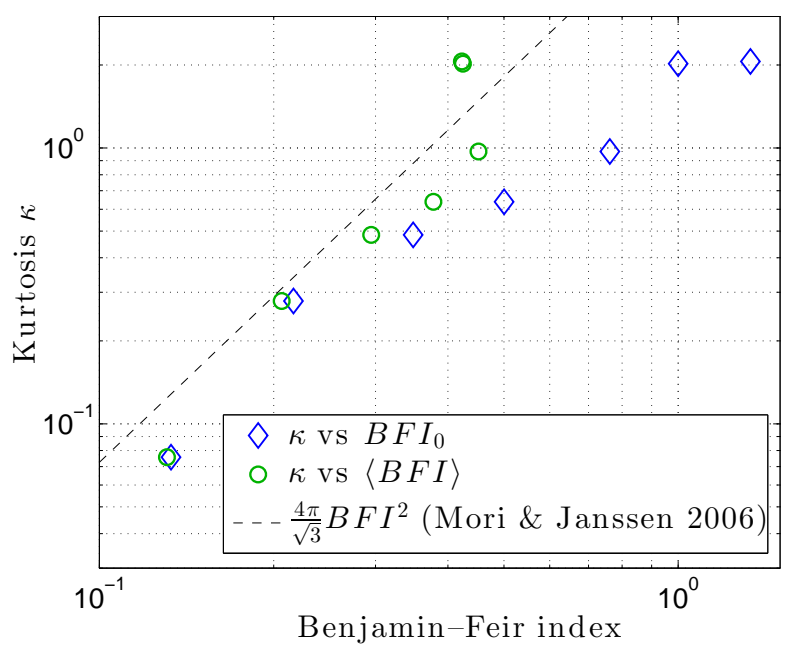

(b)

Figure 6. (a) Steady-state value of the Benjamin-Feir index, $\langle\mathrm{BFI}\rangle$, versus the initial value, $\mathrm{BFI}_{0}$, from numerical simulations. (b) Kurtosis of the surface elevation versus the BFI. The values of $\kappa$ computed from numerical simulations are plotted both as a function of the initial ( $\diamond)$ and long-time average (o) value of the BFI, respectively denoted by $\mathrm{BFI}_{0}$ and $\langle\mathrm{BFI}\rangle$. The analytical prediction by Mori $\mathcal{E}$ Janssen $[20]$ is also reported (dashed line).

obtained by MORI \& JANSsen [20] is also included in the picture. (The factor in Mori \& JANSSEN formula has been adjusted to account for the fact that we are considering the spatial, as opposed to temporal, spectrum.) Such a prediction is based on the assumption that the power spectrum is Gaussian and narrow-banded, and that the Fourier coefficients are uncorrelated in phase. Even though the assumption of a narrow-banded spectrum is clearly not legitimate at the lowest value of $\mathrm{BFI}_{0}$, where some discrepancy is therefore to be expected, we find reasonable agreement between the analytical formula and the results of numerical computations at moderate values of BFI. On the other hand, the analytical estimate fails to capture the final steep growth of $\kappa$ in the regime corresponding to very narrow-banded spectra. Such a result appears then to be a consequence of the independentphase assumption, which is expected to be unrealistic in those regimes characterized by a strong concentration of coherent structures as shown by previous studies [31, 32].

\subsection{Effect of wave steepness}

All results presented so far are obtained for the constant value of average wave steepness $s=0.04$. Understanding the effect of wave steepness is clearly a goal of primary importance, especially from the perspective of reproducing realistic oceanic conditions. Numerical simulations based on the potential flow formulation, however, are limited by the increasingly frequent occurrence of wave breaking and sharp-crested wave profiles occurring in very steep wave fields. In this section we attempt to assess the effect of $s$ on the main statistical 


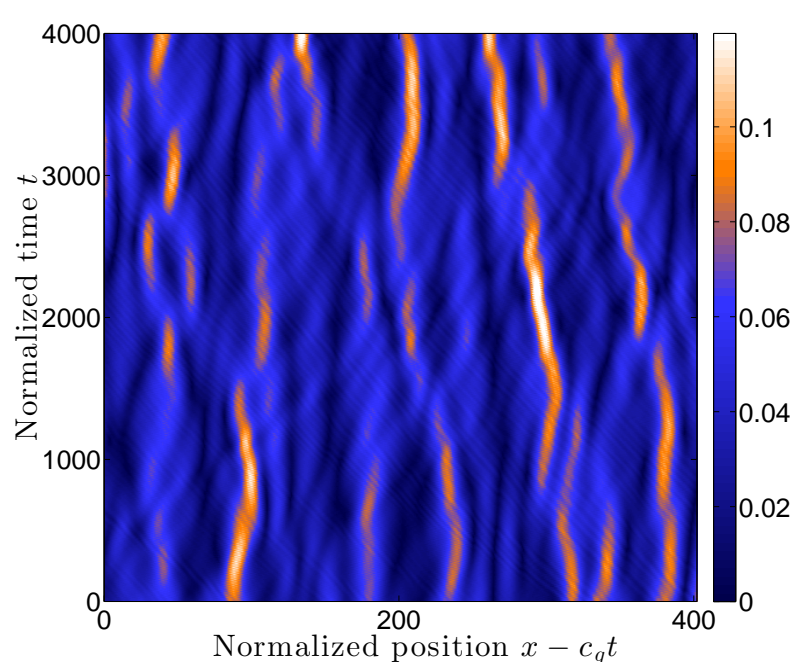

(a)

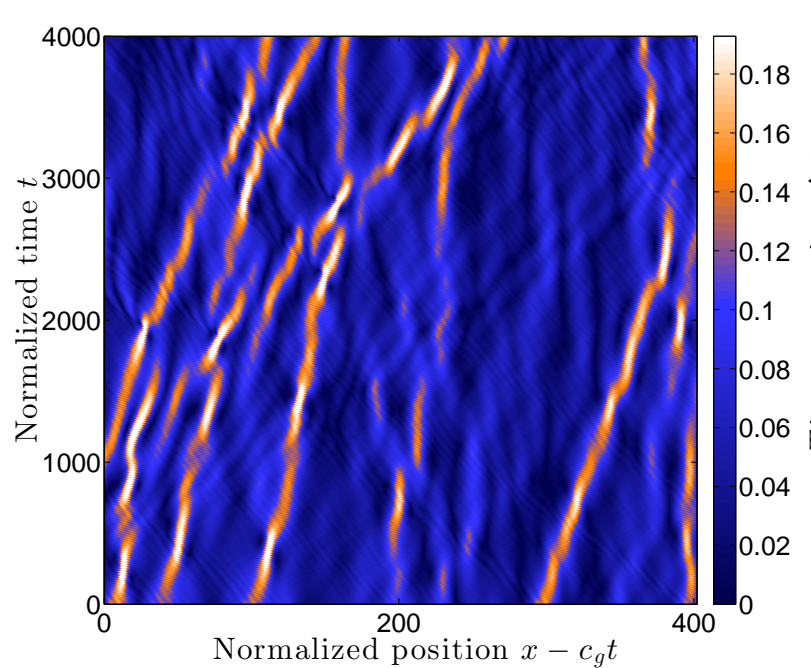

(c)

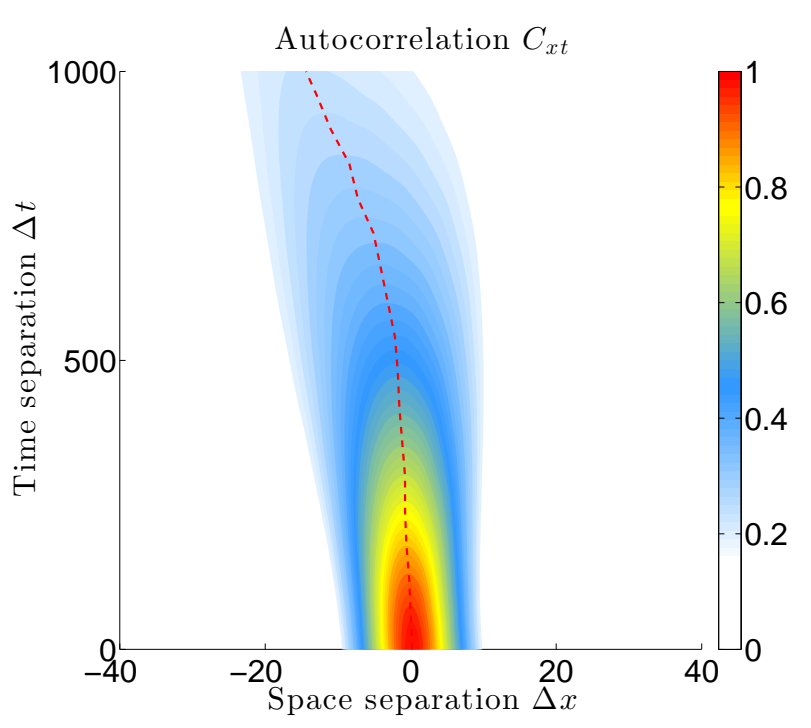

(b)

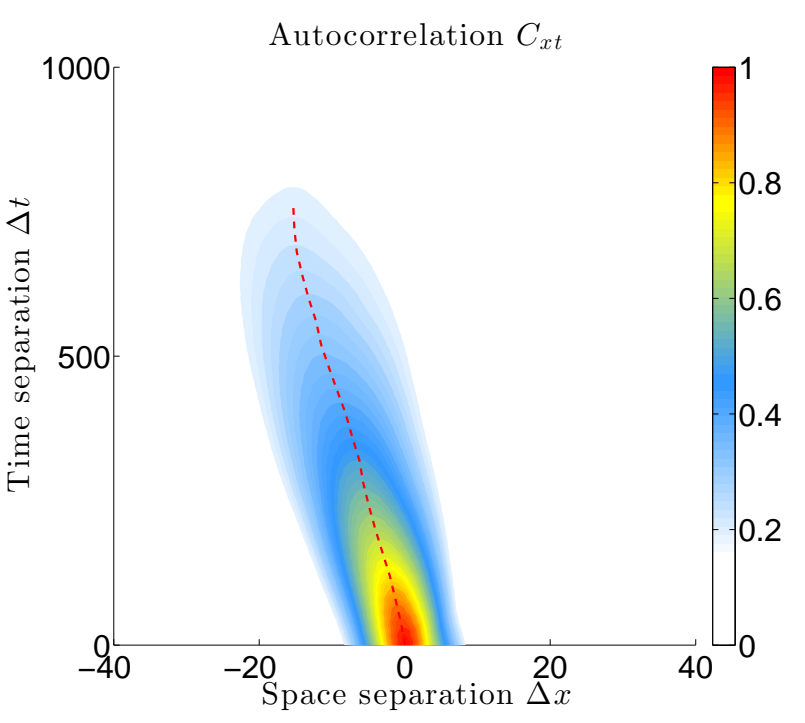

(d)

Figure 7. As figure 1, for the following parameters: $(a-b) s=0.03$, BFI $=0.375$; $(c-d) s=0.05, \mathrm{BFI}=0.625$. Dashed red curves in panels (b) and $(d)$ connect the local maxima of $C_{x t}$ along $\Delta x$ for different values of $\Delta t$.

quantities within the intrinsic limitations of the present mathematical formulation of the wave problem.

The value $s=0.04$ was found to be the highest steepness allowing to span the entire range of interest of BFI values while retaining satisfactory numerical accuracy. As it was shown in the previous sections, high values of the Benjamin-Feir index enhance the occurrence of highly steep waves, i.e., the effective degree of nonlinearity present in the flow. For those cases characterized by the highest values of such parameter, any further increase of $s$ was seen to give rise to a critical deterioration of the numerical accuracy. The simulation of steeper wave fields was found to be feasible only for moderate values of $\mathrm{BFI}_{0}$ and up to 


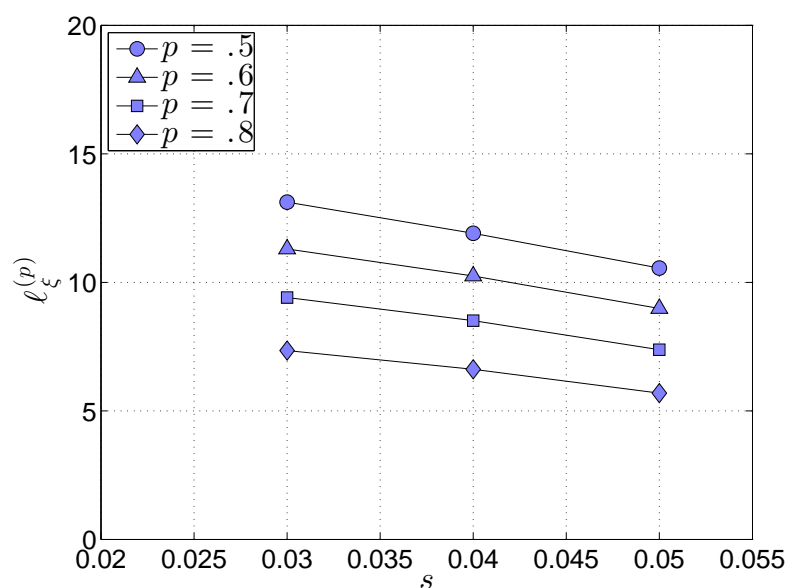

(a)

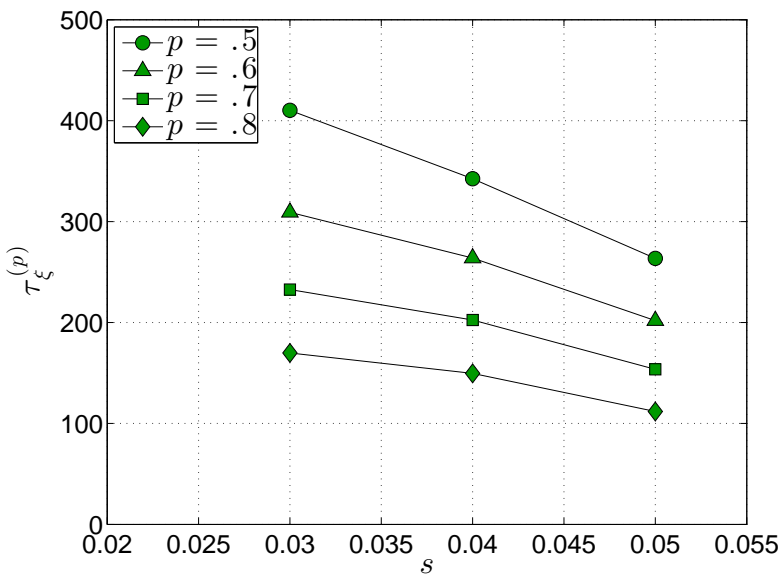

(b)

Figure 8. Spatial (a) and temporal (b) autocorrelation scales versus s from Euler numerical simulations.

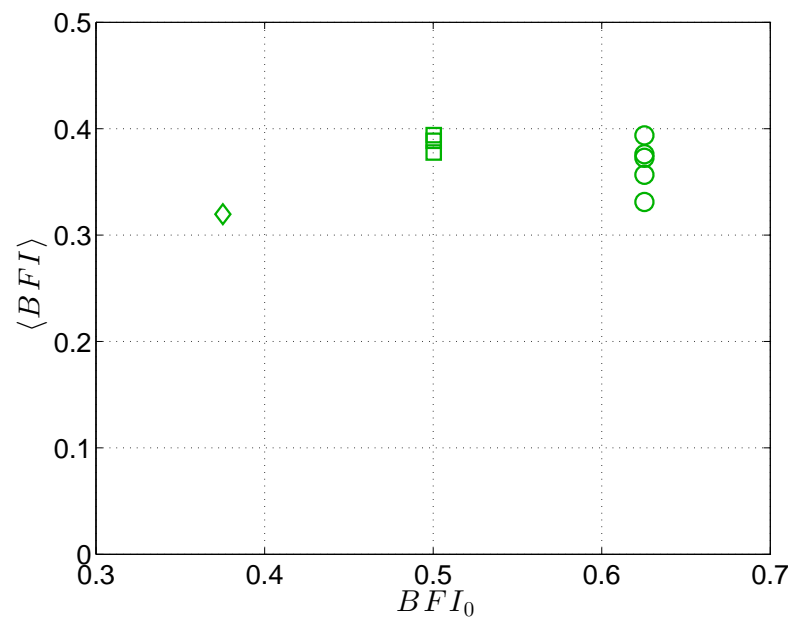

(a)

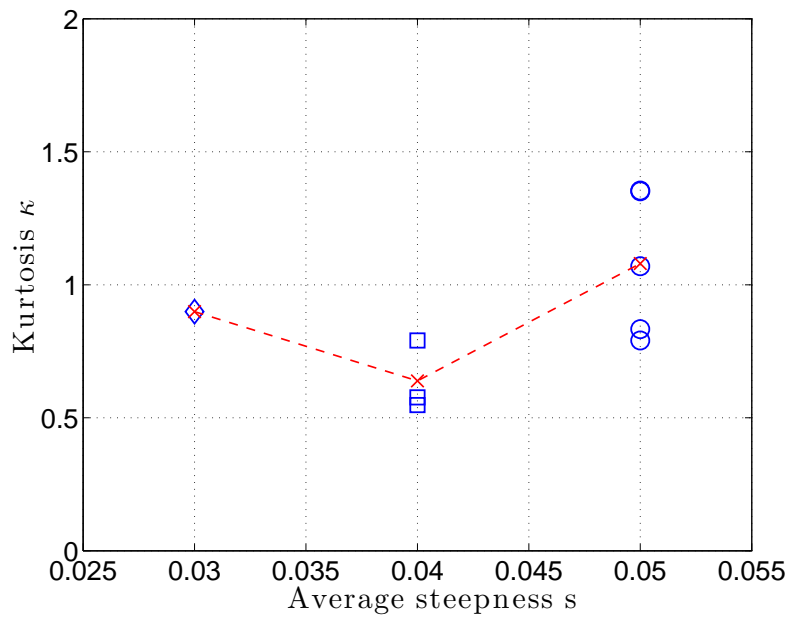

(b)

Figure 9. (a) Steady-state value of the Benjamin-Feir index, $\langle\mathrm{BFI}\rangle$, versus the initial value, $\mathrm{BFI}_{0}$, for $s=0.03,0.04,0.05$ and constant initial spectral bandwidth $\sigma_{w}=0.113$. (b) Kurtosis of the surface elevation versus $s$, for the same numerical simulations. Repeated data for the same values of $s$ correspond to different numerical simulations, of which red $(\times)$ symbols identify the mean value.

$s=0.05$. In order to better assess the dependence of wave statistics with respect to $s$, we consider also a case of a lower steepness $s=0.03$.

Typical wave field realizations for the above values of $s$ are reported in Figure 7. Beside the expected difference in the characteristic wave amplitude (note the different scales on the colorbars) we note two additional effects, which are also detected by the autocorrelation 
functions reported in the same figure. First, the steepness is seen to reduce the typical time scale of the structures present in the field, as predicted by a scaling analysis of the NLS equation. Since $\mathrm{BFI}_{0}$ increases with $s$, this result represents an inverse trend with respect to the results of the previous section, hence showing an aspect of the flow not correlated to the Benjamin-Feir index per se. Second, the mean drift speed of wave groups, already commented on in $\S 3.1$, appears to be enhanced for stronger nonlinearity. This is clearly visible in the field snapshots and detected by the corresponding autocorrelation function, which is visibly tilted in panel $(d)$. The dashed curves connecting the maxima of $C_{x t}$ along $\Delta x$ for different values of $\Delta t$, superimposed on the same plots, help to identify a characteristic slope. The reduction of the correlation length and time scales for increasing values of $s$, extracted from the same set of numerical runs considered in this section is summarized in figure 8.

Global statistics, analogue to those reported in Figure 6, are reported in Figure 9 for different values of $s$ and constant spectral bandwidth $\sigma_{w}$. The steady-state value of the Benjamin-Feir index, $\mathrm{BFI}_{0}$, is seen to settle on a subcritical value $(\approx 0.4)$ which is only marginally affected by the value of $s$. In this regard, varying the value of $\mathrm{BFI}_{0}$ by changing either $s$ or $\sigma_{w}$ does not seem to introduce any consistent difference. (Note that the run with $s=0.03$ corresponds to a subcritical Benjamin-Feir index.)

On the other hand, the effect of $s$ on the kurtosis of the surface elevation, $\kappa$, reported in Figure 9 appears to be stronger. Each value of $\kappa$ is computed from datasets resulting from individual numerical runs (characterized by $L=256 \pi$ and $T=8000$ ). This illustrates how the statistical convergence deteriorates for larger steepness, as shown by the much more scattered data distribution obtained for $s=0.05$. Despite the difficult convergence of this statistics, the trend represented by the averaged data $(\times$ symbols $)$ suggests a consistent increase of $\kappa$ in going from $s=0.04$ to $s=0.05$. In regard to the frequency of extreme events, BFI seems to represent a quite robust indicator.

\section{Conclusions}

We have presented numerical simulations of the free-surface Euler equations in deep water, with the aim to provide a joint space-time description of the coherent structures that develop in the wave field, in a setup that compares to previous experimental studies $[25,28,3,2]$. The main parameter considered in this study is the Benjamin-Feir index. We have varied such parameter by changing the spectral bandwidth while keeping the steepness constant and vice versa, even though in the latter case we have encountered more severe limitations.

The main question addressed in this study is whether the above structures can emerge spontaneously in random sea states; it is important to clarify this point before assuming confidently that coherent wave group dynamics (i.e. breathers, in the framework of the NLS equation) is the major cause of the extreme waves that occur more frequently for high values of the Benjamin-Feir index [9, 25].

We observe from our numerical runs that long-lived coherent wave groups develop progressively by increasing $\mathrm{BFI}_{0}$. For a sufficiently high $\mathrm{BFI}_{0}$, coherent structures become 
the dominant feature of the wave field. In terms of surface elevation, the maximum amplitude and the physical scales of such structures are consistent with those of Kuznetsov-Ma breathers. We also find sparse yet well-defined breather-like structures for moderate values of $\mathrm{BFI}_{0}(\approx 0.2)$, see Figure 3. At a closer look, however, the coherent structures found in this case appear to be contained in the initial condition itself, and not to be the result of a self-generating process. It is interesting to note, nonetheless, that even low-BFI wave fields can support - even though they probably cannot generate - coherent long-lived structures.

The development of the coherent wave groups visualized in figure 1 is reflected by the space-time correlation functions, which provide a quantitative measure of the associated spatial and temporal scales. For constant steepness, different growth trends with respect to BFI have been observed for the space and time scales respectively. Namely, the spatial scale was seen to dilate much slower than the temporal scale. Both quantities do not follow any simple scaling trend. When $s$ is increased, on the other hand, the trend reverses, showing that stronger nonlinearity reduces both spatial and time scales.

For those cases characterized by a supercritical $\mathrm{BFI}_{0}$, the structure of the wave field persists after $\mathrm{BFI}(\mathrm{t})$ has settled on a subcritical value. This observation stands at odds with the idea that rogue waves are a transitional feature of the wave field associated with developing Benjamin-Feir instability. This particular aspect was mostly hinted by experimental data, but it is better clarified by numerical simulations, as the effective time span observable in laboratory experiments is limited by the length of the wave tank. Transient effects are hardly removed, as pointed out by SLunyaev \& Sergeeva [32], even in state-of-the-art facilities (cf. § 3).

\section{Acknowledgments}

This publication has emanated from research conducted with the financial support of European Research Council under the research project ERC-2011-AdG 290562-MULTIWAVE and Science Foundation Ireland under Grant Number SFI/12/ERC/E2227.

\section{References}

[1] K. Al-Salem, W. Al-Nassar, and M. A. Tayfun. Risk analysis for capsizing of small vessels. Ocean Engineering, 33:788-797, 2006. 2

[2] A. Chabchoub, N. N. Akhmediev, and N. P. Hoffmann. Experimental study of spatiotemporally localized surface gravity water waves. Phys. Rev. E, 86:016311, 2012. 2, 15

[3] A. Chabchoub, N. P. Hoffmann, and N. N. Akhmediev. Rogue wave observation in a water wave tank. Phys. Rev. Lett., 106:204502, 2011. 2, 15

[4] W. Choi and R. Camassa. Exact Evolution Equations for Surface Waves. J. Eng. Mech., 125(7):756, 1999. 4

[5] F. Dias, T. J. Bridges, and J. M. Dudley. Environmental hazards. The fluid dynamics and geophysics of extreme events. In Lecture Notes Series. World Scientific Publishing. 2011. 2

[6] A. I. Dyachenko and V. E. Zakharov. Modulation instability of stokes waves $\rightarrow$ freak wave. JETP Lett., 81(6):255-259, 2005. 3

[7] A. I. Dyachenko, V. E. Zakharov, and E. A. Kuznetsov. Nonlinear dynamics of the free surface of an ideal fluid. Plasma Physics Reports, 22(10):829-840, 1996. 4 
[8] K. B. Dysthe, H. E. Krogstad, and P. Muller. Oceanic rogue waves. Ann. Rev. Fluid Mech., 40(40):287310, 2008. 2

[9] K. B. Dysthe and K. Trulsen. Note on breather type solutions of the NLS as models for freak-waves. Physica Scripta, T82:48-52, 1999. 2, 15

[10] F. Fedele. On wave groups in a Gaussian sea. Ocean Engineering, 33(17-18):2225-2239, Dec. 2006. 6

[11] G. Genty, C. M. de Sterke, O. Bang, F. Dias, N. N. Akhmediev, and J. M. Dudley. Collisions and turbulence in optical rogue wave formation. Phys. Lett. A, 374:989-996, 2010. 2, 6

[12] O. Gramstad and K. Trulsen. Influence of crest and group length on the occurrence of freak waves. $J$. Fluid Mech., 582:463-472, 2007. 3

[13] K. L. Henderson, D. H. Peregrine, and J. W. Dold. Unsteady water wave modulation: fully nonlinear solutions and comparison with the nonlinear Schrödinger equation. Wave Motion, 29:341-361, 1999. 2,9

[14] N. E. Huang, Z. Shen, and S. R. Long. A new view of nonlinear water waves: the Hilbert spectrum. Ann. Rev. Fluid Mech., 31:417-457, 1999. 5, 6

[15] P. A. E. M. Janssen. Nonlinear four-wave interactions and freak waves. Phys. Oceanogr., 33:863-884, 2003. 3,4

[16] C. Kharif and E. Pelinovsky. Physical mechanisms of the rogue wave phenomenon. Eur. J. Mech. B/Fluids, 22:603-634, 2003. 2

[17] B. Kibler, J. Fatome, C. Finot, G. Millot, F. Dias, G. Genty, N. Akhmediev, and J. M. Dudley. The Peregrine soliton in nonlinear fibre optics. Nature Physics, 6:790-795, 2010. 2, 9

[18] B. Kibler, J. Fatome, C. Finot, G. Millot, G. Genty, B. Wetzel, and N. N. Akhmediev. Observation of Kuznetsov-Ma soliton dynamics in optical fibre. Sci. Rep., 2(463), 2012. 2

[19] P. Milewski, J.-M. Vanden-Broeck, and Z. Wang. Dynamics of steep two-dimensional gravity-capillary solitary waves. J. Fluid Mech., 664:466-477, 2010. 4

[20] N. Mori and P. A. E. M. Janssen. On Kurtosis and occurrence probability of freak waves. J. Phys. Oceanogr., 36:1471-1483, 2006. 11, 12

[21] I. Nikolkina and I. Didenkulova. Rogue waves in 2006 - 2010. Nat. Hazards Earth Syst. Sci., 11:29132924, 2011. 2

[22] L. O'Brien, J. M. Dudley, and F. Dias. Extreme wave events in Ireland: 14680 BP-2012. Natural Hazards and Earth System Science, 13(3):625-648, Mar. 2013. 2

[23] M. Onorato, A. Osborne, R. Fedele, and M. Serio. Landau damping and coherent structures in narrowbanded 1+1 deep water gravity waves. Phys. Rev. E, 67(4):046305, Apr. 2003. 2

[24] M. Onorato, A. R. Osborne, M. Serio, and S. Bertone. Freak waves in random oceanic sea states. Phys. Rev. Lett., 86(25):5831-5834, 2001. 2

[25] M. Onorato, A. R. Osborne, M. Serio, L. Cavalieri, C. Brandini, and C. T. Stansberg. Observation of strongly non-gaussian statistics for random sea surface gravity waves in wave flume experiments. Phys. Rev. E, 70:067302, 2004. 3, 5, 10, 15

[26] A. R. Osborne, M. Onorato, and M. Serio. The nonlinear dynamics of rogue waves and holes in deep-water gravity wave trains. Phys. Lett. A, 275(5-6):386-393, Oct. 2000. 2

[27] A. N. Pushkarev and V. E. Zakharov. Quasibreathers in MMT model. Phys. D, In Press, 2013. 2

[28] L. Shemer, A. Sergeeva, and D. Liberzon. Effect of the initial spectrum on the spatial evolution of statistics of unidirectional nonlinear random waves. J. Geophys. Res., 115(C12):C12039, Dec. 2010. 3, 5,15

[29] L. Shemer, A. Sergeeva, and A. Slunyaev. Applicability of envelope model equations for simulation of narrow-spectrum unidirectional random wave field evolution: Experimental validation. Phys. Fluids, 22(1):016601, 2010. 3

[30] P. K. Shukla, I. Kourakis, B. Eliasson, M. Marklund, and L. Stenflo. Instability and evolution of nonlinearly interacting water waves. Phys. Rev. Lett., 97:094501, 2006. 2

[31] A. Slunyaev. Freak wave events and the wave phase coherence. Eur. Phys. J. Special Topics, 185:67-80, 2010. 12 
[32] A. Slunyaev and A. Sergeeva. Stochastic simulations of unidirectional intense waves in deep water applied to turbulence. JETP Lett., 94(10):843-851, 2011. 3, 12, 16

[33] H. Socquet-Juglard, K. B. Dysthe, K. Trulsen, H. E. Krogstad, and J. Liu. Probability distributions of surface gravity waves during spectral changes. J. Fluid Mech., 542:195-216, 2005. 3

[34] H. Tamura, T. Waseda, and Y. Miyazawa. Freakish sea state and swell-windsea coupling: Numerical study of the Suwa - Maru incident. Geophys. Res. Lett., 36(1):L01607, Jan. 2009. 3

[35] M. A. Tayfun. Narrow-band nonlinear sea waves. J. Geophys. Res., 85:1548-1552, 1980. 10

[36] A. Toffoli and E. M. Bitner-Gregersen. Extreme and rogue waves in directional wave fields. Open Ocean Eng. J., 4:24-33, 2011. 3

[37] V. E. Zakharov and R. V. Shamin. Probability of the occurrence of freak waves. JETP Lett., 91(2):6265, 2010. 3

University College Dublin, School of Mathematical Sciences, Belfield, Dublin 4, IreLAND

E-mail address: claudio.viotti@ucd.ie

URL: http://www.ercmultiwave.eu/team/view/claudio-viotti/

University College Dublin, School of Mathematical Sciences, Belfield, Dublin 4, Ireland and LAMA, UMR 5127 CNRS, Université de Savoie, Campus Scientifique, 73376 Le Bourget-du-Lac Cedex, France

E-mail address: Denys.Dutykh@ucd.ie

$U R L:$ http://www.denys-dutykh.com/

Département d'Optique P.M. Duffieux, Université de Franche-Comté, Institut FemtOST CNRS UMS 6174, Besançon, France

E-mail address: John.Dudley@univ-fcomte.fr

CMla, ens Cachan, CNRs, 61 Avenue du Président Wilson, F-94230 Cachan, France and University College Dublin, School of Mathematical Sciences, Belfield, Dublin 4, IRELAND

E-mail address: Frederic.Dias@ucd.ie 